\title{
Accumulation of Somatic Mutations in TP53 in Gastric Epithelium With Helicobacter pylori Infection.
}

\section{$\operatorname{AUTHOR}(\mathrm{S}):$}

Shimizu, Takahiro; Marusawa, Hiroyuki; Matsumoto, Yuko; Inuzuka, Tadashi; Ikeda, Atsuyuki; Fujii, Yosuke;

Minamiguchi, Sachiko; ... Sakai, Yoshiharu; Crabtree, Jean E; Chiba, Tsutomu

\section{CITATION:}

Shimizu, Takahiro ... [et al]. Accumulation of Somatic Mutations in TP53 in Gastric Epithelium With Helicobacter pylori Infection.. Gastroenterology 2014, 147(2): 407-417.e3

\section{ISSUE DATE:}

2014-08

URL:

http://hdl.handle.net/2433/189431

\section{RIGHT:}

(C) 2014 AGA Institute. Published by Elsevier Inc.; This is not the

published version. Please cite only the published version.; この論文は

出版社版でありません。引用の際には出版社版をご確認ご利用くださ い。 


\title{
Accumulation of Somatic Mutations in TP53 in Gastric Epithelium
}

\author{
with Helicobacter pylori infection.
}

Takahiro Shimizu' ${ }^{1}$, Hiroyuki Marusawa ${ }^{1}$, Yuko Matsumoto ${ }^{1}$, Tadashi Inuzuka ${ }^{1}$, Atsuyuki Ikeda ${ }^{1}$, Yosuke Fujii ${ }^{1}$, Sachiko Minamiguchi ${ }^{2}$, Shin'ichi Miyamoto ${ }^{1}$, Tadayuki Kou $^{3}$, Yoshiharu Sakai ${ }^{4}$, Jean E Crabtree ${ }^{5}$ and Tsutomu Chiba ${ }^{1}$

1. Department of Gastroenterology and Hepatology, Graduate School of Medicine, Kyoto University, Kyoto, Japan

2. Department of Diagnostic Pathology, Graduate School of Medicine, Kyoto University, Kyoto, Japan

3. Digestive Disease Center, The Tazuke Kofukai Medical Research Institute, Kitano Hospital, Osaka, Japan

4. Department of Gastrointestinal Surgery, Graduate School of Medicine, Kyoto University, Kyoto, Japan

5. Leeds Institute Molecular Medicine, University of Leeds, Leeds, United Kingdom

Corresponding \& Reprint Author: Hiroyuki Marusawa at:

Department of Gastroenterology and Hepatology, Graduate School of Medicine, Kyoto University, 54 Kawahara-cho, Shogoin, Sakyo-ku, Kyoto 606-8507, Japan

Phone; +81-75-751-4319, Fax; +81-75-751-4303

E-mail; maru@kuhp.kyoto-u.ac.jp

Short Title: Mutations in H. pylori (+) inflamed gastric mucosa.

Grant Support: This work was supported by Japan Society for the Promotion of Science (JSPS) KAKENHI (25130706, 24229005 and 24659363), and Grants-in-aid for Scientific Research on Innovative Areas from the Ministry of Education, Culture, Sports Science and Technology 
(MEXT) of Japan.

Abbreviations: AID: activation-induced cytidine deaminase, H. pylori: Helicobacter pylori, Hupki: human TP53 knock-in, indel: insertion and deletion, MSI: microsatellite instability, MSS: microsatellite stability, SNV: single nucleotide variant

Disclosures: The authors have no conflicts of interest.

Data Profiling: Sequence reads were deposited in the DNA Data Bank of Japan Sequence Read Archive (http://trace.ddbj.nig.ac.jp/dra/index_e.shtml) under accession numbers DRA001035.

Author contributions: T.S. conducted all the experiments and contributed to manuscript preparation. H.M. designed the study, supervised the project and contributed to manuscript preparation. Y.M., T.I. and A.I. performed deep sequencing analyses. Y.F. performed the bioinformatics data analysis. S.M. performed histological examination. S.M., T.K. and Y.S. collected human samples. J.E.C. and T.C. supervised the project. 


\begin{abstract}
Background \& Aims: Helicobacter pylori infection is a risk factor for gastric cancer. To explore the genetic basis of gastric cancer that develops in inflamed gastric mucosa, we investigated genetic aberrations that latently accumulate in non-tumorous gastric epithelium with $H$ pylori infection.

Methods: We performed whole exome sequencing of gastric tumors, non-cancerous tissues with gastritis, and peripheral lymphocytes from 5 patients. We performed additional deep-sequencing analyses of selected tumor-related genes using 34 gastritis mucosal samples from patients with or without gastric cancer. We also performed deep sequencing analyses of gastric mucosal tissues from mice that express transgenic human TP53 and constitutively express activation-induced cytidine deaminase (AICDA or AID) (Hupki/AID-Tg mice).
\end{abstract}

Results: Whole-exome sequencing revealed that somatic mutations accumulated in various genes in inflamed gastric tissues. Additional deep sequencing analyses of tissues from regions of gastritis confirmed non-synonymous low-abundance mutations in TP53 in 15 cases (44.1\%) and ARIDIA in 5 cases $(14.7 \%)$. The mutations that accumulated in gastric mucosal tissues with $H$ pylori-induced gastritis, as well as gastric tumors, were predominantly $\mathrm{C}: \mathrm{G}>\mathrm{T}$ :A transitions in GpC $\mathrm{pX}$ motifs - a marker of cytidine deamination induced by AID. Constitutive expression of AID in the gastric mucosa of mice led to mutations in the human TP53, at amino acid coding positions identical to those detected in human gastric cancers.

Conclusions: Studies of gastric tumors and tissues from humans and mice indicate that somatic mutations accumulate in various genes in gastric mucosal tissues with $H$ pylori infection. Increased cytidine deaminase activity in these tissues appears to promote the accumulation of these mutations and might promote gastric carcinogenesis in patients with H pylori infection.

Keywords: stomach cancer; somatic hypermutation; pathogenesis; bacteria 


\section{Introduction}

The recent introduction of deep sequencing technology, capable of detecting sequences of hundreds of thousands of individual molecules, is rapidly changing the landscape of cancer genome research. Comprehensive genome analyses using the deep sequencing technique have clarified that each tumor cell has numerous nucleotide alterations ${ }^{1}$. Among the genetic aberrations, driver mutations are thought to contribute to tumorigenesis by conferring a selective advantage to the cell. On the other hand, passenger mutations are defined as those that do not alter fitness but occur in a cell that coincidently, or subsequently, acquired a driver mutation ${ }^{2}$. Recent reports demonstrated that human gastric cancer has approximately 66 to 212 mutations in coding regions, and only a few of them are considered driver mutations ${ }^{3-6}$. Among them, mutations in the tumor suppressor TP53 gene are the most common driver mutations in human gastric cancer tissues. A second common putative driver mutation was determined in the chromatin remodeling complexes gene, $A R I D I A^{3}$. It remains unclear, however, when and how those driver mutations emerge in the gastric epithelium during tumorigenesis.

Epidemiologic studies revealed that patients with Helicobacter pylori ( $H$ pylori) infection have a significantly increased risk of gastric cancer compared to $H$ pylori-negative individuals ${ }^{7,8}$. The more prevalent form of gastric adenocarcinoma frequently progresses through a series of histologic steps that are initiated by chronic gastritis, which then leads to atrophic gastritis and intestinal metaplasia, and finally to dysplasia and adenocarcinoma in individuals with $H$ pylori infection ${ }^{9}$. Multicentric tumor development characterized in $H$ pylori-associated gastric cancer ${ }^{10}$ suggests that the $H$ pylori-infected stomach could include epithelial cells and/or intestinal metaplasia with genetic damage that possess high susceptibility to tumor development ${ }^{11}$. Consistent with these findings, animal experiments using Big Blue mice revealed a high mutation frequency in the $H$ pylori-infected gastric mucosa ${ }^{12}$, which are increased in 
Helicobacter-infected Big Blue-p53 hemizygous mice ${ }^{13}$. The precise mechanism for the emergence of genetic alterations in $H$ pylori-infected gastric mucosa, however, has not been elucidated. We recently demonstrated that $H$ pylori infection and the resultant chronic inflammation with mononuclear cell infiltration induces genetic alterations, including somatic mutations and chromosomal aberrations, in gastric epithelial cells through the expression of a DNA-mutator enzyme, activation-induced cytidine deaminase (AID $)^{14-17}$. In vitro studies revealed that $H$ pylori infection elicited the ectopic expression of AID in association with a high mutation frequency of the TP53 genome sequences ${ }^{14}$. To date, however, there has not been enough evidence showing that AID expression in gastric mucosa in vivo causes the accumulation of driver mutations in the human TP53 sequences required for malignant transformation.

To clarify the mechanisms underlying the contribution of inflammation to carcinogenesis, it is important to identify the genetic alterations that occur in the inflamed epithelium before the onset of tumorigenesis. The main obstacle to detecting latently accumulated genetic alterations in non-tumorous inflamed tissue is the diversity and low frequency of the mutated genes ${ }^{18}$. In the present study, to unveil the landscape of accumulated genetic aberrations in chronically inflamed gastric epithelium, we performed whole exome sequencing and additional deep sequencing of the inflamed gastric mucosa with chronic $H$ pylori infection. Furthermore, to confirm whether constitutive AID expression in vivo contributes to the emergence of the TP53 mutations, we determined the mutation profile of the TP53 gene in the AID-expressing gastric mucosa of the human TP53 knock-in mouse model. 


\section{Materials and methods}

\section{Whole exome capture and massively-parallel sequencing.}

Massively-parallel sequencing was performed as described previously ${ }^{19}{ }^{20}$. Fragmented DNA (>5 $\mu \mathrm{g}$ ) was used to prepare each DNA sequencing library. The DNA libraries were prepared according to the instructions provided with the Illumina Preparation Kit (Illumina, San Diego, CA). Whole exome sequence capture was then performed using SeqCap EZ Human Exome Library v2.0 (Roche, Madison, WI) according to the manufacturer's instructions. Cluster generation was performed on the Illumina cluster station using their TruSeq PE Cluster Kit v5. Paired-end sequencing for $2 \times 76$ base pair (bp) reads was performed on the Illumina Genome Analyzer IIx using their SBS Kits v5. Data collection and base-calling were performed using SCS v2.9/RTA 1.9 and resultant data files were converted to the FASTQ format.

Sequencing data were analyzed with NextGENe 2nd Generation Sequence Analysis Software v2.2.0 (SoftGenetics, State College, PA). We identified somatic mutations by the strict variant filtering process (Supplementary Figure 1 and Supplementary materials and methods). To validate somatic mutations, we performed PCR-based deep-sequencing using a different deep-sequencing platform (the GS Junior System, Roche) on the non-silent mutations detected by whole exome sequencing, according to the validation protocol reported in the recent study ${ }^{21}$. We estimated that the reliabilities of whole exome sequencing were $88.6 \%$ for somatic mutations detected in the tumor tissues and $86.4 \%$ for somatic mutations detected in the non-tumor tissues (data not shown). Primer sequences are shown in Supplementary Table 1.

\section{Deep sequencing on selected genes.}

The mutational status of TP53, ARID1A, MLL2, SUV39H1, CTNNB1, PIK3CA, and TP53 cDNA was investigated by high-coverage sequencing analyses. Target regions were designed within the range from 330 to $520 \mathrm{bp}$. The primer sequences are described in Supplementary 
Table 2. Each region was PCR-amplified with Phusion High-Fidelity PCR Enzyme (FINZYMES, Espoo, Finland), purified by gel-extraction methods. Five hundred nanograms of each sample was dA-tailed and ligated with adaptors containing tag sequences, followed by emulsion PCR and sequencing using the GS Junior System (Roche) according to the manufacturer's protocol.

Deep sequencing data were analyzed with NextGENe software. We identified low-abundance somatic mutations by the strict variant filtering process (Supplementary Figure 2 and Supplementary materials and methods). Candidate low-abundance mutations were validated by repeated deep sequencing using independent amplicons derived from the same samples.

\section{Tissue specimens and DNA extraction.}

Animal Experiments.

Whole exome sequencing analysis and variant filtering.

Deep sequencing analysis and variant filtering.

Functional prediction analysis and pathway analysis.

\section{Statistical analysis.}

These procedures and information are described in the Supplementary Materials and Methods. 


\section{Results}

\section{Landscape of genetic alterations that accumulated in $\mathrm{H}$ pylori-associated gastric cancers.}

To overview the landscape of genetic alterations that accumulated in both gastric cancer and the adjacent background inflamed mucosa with $H$ pylori infection, we performed whole exome sequencing of 5 matched pairs of gastric cancer and non-cancerous gastritis tissues in patients with $H$ pylori infection. To subtract the normal variants of each individual from the somatic mutations, we also determined the whole exome sequences of the matched peripheral lymphocytes in each patient. Microsatellite analysis revealed that one gastric cancer had microsatellite instability (MSI) and the remaining four tumors had the characteristics of microsatellite stability (MSS). All background gastric mucosa had the features of chronic gastritis with mucosal atrophy and intestinal metaplasia and none of the gastric specimens included dysplastic lesions (Supplementary Table 3).

We targeted the whole exons of approximately 20,000 genes, sequenced $3.33 \mathrm{~Gb}$ on average for each sample, and achieved 41.2-fold coverage per samples as the mean coverage of each base in the target regions (Supplementary Table 4). According to the variant filtering process (Supplementary Figure 1), we identified single nucleotide variants (SNVs), short insertions and deletions (indels) in cancer tissues as well as in the non-cancerous gastritis mucosa.

We identified 892 somatic mutations in 793 genes in the 5 gastric cancer tissues. The identified mutations included 654 non-silent mutations (552 missense, 25 nonsense, 1 stop codon loss, 11 essential splice site mutations, and 65 indels) and 238 synonymous mutations (Supplementary Tables 5, 6). The number of non-silent mutations in the MSI gastric cancer sample was significantly higher than those of any of the MSS tumor samples (Figure 1A). In addition, the MSI tumor also had a large number of indels, while the MSS tumors had only a few indels (Figure 1C, Supplementary Table 5). Furthermore, the mutation frequency at each 
nucleotide position in the MSI tumor tended to be higher than those in the MSS tumors (Figure 1B). These findings indicated that MSI gastric cancers have enhanced genetic aberrations compared with MSS tumors.

\section{Mutation profiles of MSS and MSI gastric cancer tissues.}

Among the mutated genes identified, 26 genes were recurrently mutated as non-synonymous mutations or indels in two or more gastric cancers (Table 1). These genes included TP53 (mutated in 3 of 5 cases), and ARIDIA (mutated in 2 of 5 cases), mutations of which were mutually exclusive $\mathrm{e}^{3,4}$. All of the TP53 mutations were present as a type of nucleotide substitution in three MSS tumor tissues, whereas the ARIDIA mutations were detected as deletions in the one remaining MSS and in one MSI gastric cancer. Pathway analyses in the five gastric cancers revealed that cell adhesion ${ }^{4}$ was the most significantly enriched biological process, followed by metabolic processes, endocytosis and extracellular structure organization (Supplementary Table 7).

Recent studies showed that the pattern of nucleotide alterations could provide a clue to determine the molecular process underlying mutation occurrence ${ }^{22-27}$. We first confirmed that over half of the SNVs that accumulated in the exome sequences of gastric cancer tissues were enriched with a $\mathrm{C}: \mathrm{G}>\mathrm{T}$ :A transition $(\mathrm{p}<0.01$ by ANOVA, Figure $1 \mathrm{C})$. The prevalence of these $\mathrm{C}: \mathrm{G}>\mathrm{T}: \mathrm{A}$ transitions in the MSI gastric cancer was substantially higher than that in the MSS tumors $(65.1 \%$ vs. $48.9 \%$, respectively). These mutational signatures suggested that cytidine to uracil deamination is deeply implicated in the mutational process underlying $H$ pylori-associated gastric carcinogenesis. Among the cytidine deaminase family proteins, AID exhibits a strong preference for deaminating $C$ residues flanked by a 5 '-purine $(G \text { or } A)^{22,28,29}$. In contrast, APOBEC3 family enzymes and APOBEC1 favor $\mathrm{C}$ residues flanked by $5^{\prime}-\mathrm{T}^{30,31}$. Therefore, we 
further evaluated the sequence characteristics flanking the mutated $\mathrm{C}$ residues in tumor tissues. Consistent with the previous report ${ }^{5}$, we found that the $\mathrm{Gp} \underline{\mathrm{CpX}}(\underline{\mathrm{C}}$ is the mutated nucleotide and

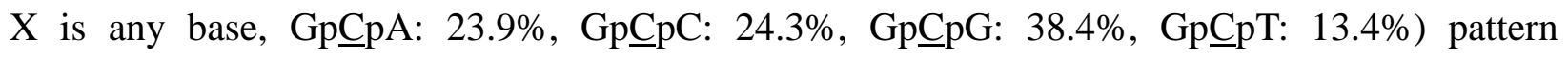
dominated across all of the tissues examined ( $\mathrm{p}<0.01$ by ANOVA), followed by $\mathrm{Cp} \underline{\mathrm{C} X}$ and ApCpX (Figure 1D), suggesting that deamination achieved by AID activity is likely to be involved in the mutational signature of $H$ pylori-associated gastric cancers.

\section{Overview of genetic alterations that accumulated in non-cancerous gastritis mucosa with $\boldsymbol{H}$ pylori infection.}

Whole exome sequencing clarified that various mutations were also present in the non-cancerous $H$ pylori-infected gastritis mucosa. All of the mutations detected in the non-tumorous gastric mucosa were SNVs and no indels were observed in any of the non-tumorous gastritis tissues. The mean number of mutated genes in the non-tumorous tissues was 28.4 per sample, which was lower than that in the matched tumor tissues of all 5 patients (Supplementary Tables 5, 8). In addition, the mutation frequency at each nucleotide position represented less than $20 \%$ of the total reads of the majority of the altered genes in non-tumorous gastric mucosa (Figure 1B), indicating that the mutation frequency at each nucleotide position tended to be lower in the non-cancerous tissues than in the cancer tissues. The total number of mutated genes and mutant allele frequencies in the non-tumorous tissues did not significantly differ between the patient with MSI and those with MSS cancers (Figure 1A, 1B, Supplementary Table 5).

Among the 142 mutations identified in the 5 non-tumorous gastritis tissues, 91 mutations $(64.1 \%)$ were non-silent. Sorting Intolerant From Tolerant (SIFT) functional impact predictions ${ }^{32}$ revealed that the mean percentages of somatic mutations predicted to be "damaging" or 
frame-shift, nonsense and splice site mutations in non-tumorous gastritis and cancer tissues were $29.6 \%$ and $44.7 \%$, respectively ( $p<0.01$ by $\chi^{2}$ test), suggesting that the SNVs that accumulated in the non-tumorous tissues more frequently included "passenger" mutations with less functional significance than those in the tumor tissues (data not shown). Pathway analyses revealed that transcription-related genes, such as $M L L 2$ and SUV39H1 that are involved in chromatin or histone modification, were frequently altered in non-tumorous gastritis tissues (Supplementary Table 9). Whole exome sequencing also revealed 25 genes that were commonly mutated in both the tumor and the matched background gastritis mucosa of the same patients (Supplementary Tables 10 and 11). Among them, 16 genes acquired non-synonymous mutations and Sanger sequencing confirmed that those SNVs were not detected in the matched control lymphocytes, while the biological role of these mutated genes remains unclear.

\section{Establishment of deep sequencing platform for detecting low-abundance mutations in tumor-related genes.}

Whole exome sequencing clarified that somatic mutations accumulated in various genes in $H$ pylori-infected gastritis mucosa, while the majority of the mutated genes detected were supposed to be "passenger" mutations. Those findings prompted us to examine whether somatic mutations latently accumulate in "driver" genes that could contribute to gastric tumorigenesis, and thus deep sequencing analysis of the selected amplicons of tumor-related genes were applied to the non-tumorous gastric mucosa and the matched peripheral lymphocytes from $H$ pylori $(+)$ patients with and without gastric cancers.

First, we conducted control experiments to validate the efficacy and error rates in deep sequencing using a plasmid encoding the TP53 cDNA sequence as a template. The deep sequencing platform provided us the TP53 sequences derived from the plasmids with a mean 
coverage of 4024 at each nucleotide site and the error rate per nucleotide position was below $0.07 \%$. To estimate the accuracy of detecting nucleotide alterations using reads filtered by mean base quality and mapping quality, we introduced expression plasmids with a single point mutation within this wild-type sequence with a ratio of $1 \%(1: 100), 0.1 \%(1: 1000)$, or 0.01\%(1:10000). Duplicate control experiments revealed that mutations present at an input ratio of $0.1 \%$ were detected ranging between $0.11 \%$ and $0.12 \%$ (Supplementary Table 12). Based on these results, the limit of mutant allele frequencies detectable by this deep sequencing was considered to be more than $0.1 \%$ of the total reads. Finally, to exclude the errors during the sequencing process, repeated experiments were performed and we determined that nucleotide alterations validated by at least more than two different deep sequencings were low-abundance somatic mutations.

\section{Somatic mutations accumulated in tumor-related genes in $H$ pylori infection-related gastritis mucosa.}

We performed deep sequencing on the selected amplicons of tumor-related genes in $H$ pylori-related gastritis mucosa in 28 patients with gastric cancer and 6 patients without gastric cancer (Supplementary Table 13). Consistent with the previous studies ${ }^{3,4}$, our whole exome sequencing on 5 tumor tissues identified that TP53 and ARIDIA were frequently mutated in gastric cancer tissues. In addition, the previous studies clarified that CTNNB1 and PIK3CA are also frequently mutated in gastric cancer tissues ${ }^{3,4}$. Based on those findings, we selected these four genes (TP53, ARIDIA, CTNNB1 and PIK3CA) for further deep-sequencing analyses. In addition, we targeted $M L L 2$ and $S U V 39 H 1$, which were identified by current whole exome sequencing in non-cancerous gastritis tissues. The mean coverage ranged from 2319 to 12,934 fold at each targeted gene. 
We first verified that no nucleotide alterations were detected in normal gastric mucosa without H pylori infection or any lymphocytes of the enrolled patients. Deep sequencing on 34 gastritis mucosa revealed that a lot of non-synonymous mutations were present at a very low frequency $(0.11-1.12 \%)$ in various genes $\left(\mathrm{p}<0.05\right.$ by $\chi^{2}$ test $)$. We validated the results by repeated deep sequencings and confirmed that $83.7 \%$ of the nucleotide changes detected by the initial analyses were also detected by the independent deep sequencing analyses (Supplementary Table 14). In the gastritis mucosa of 28 patients with gastric cancer, we identified non-synonymous low-abundance mutations in 13 nucleotide positions of TP53 in 11 cases, 6 positions of ARIDIA in 4 cases, and 3 positions of MLL2 in 2 cases, while no mutations were detected in CTNNB1, PIK3CA, and SUV39H1 (Table 2). In addition, in the gastritis mucosa of 6 patients without gastric cancer, non-synonymous low-abundance mutations were detected in 6 positions of TP53 in 4 cases and in 1 position of $A R I D I A$ in 1 case (Table 3).

Mutational status in non-cancerous gastritis mucosa was not significantly associated with the stage, differentiation, MSI status, or mutational status of the corresponding gastric cancers (data not shown). Regarding the mutation signature in non-tumorous gastritis mucosa, deaminating $\mathrm{C}$ residues flanked by a 5'-purine (G or $A$ ) were strongly preferred, similar to that in gastric cancer tissues. Indeed, 15 of 19 (78.9\%) low-abundance TP53 mutations in $H$ pylori-related gastritis mucosa with or without gastric cancer were $\mathrm{C}: \mathrm{G}>\mathrm{T}$ :A transitions $\left(\mathrm{p}<0.01\right.$ by $\chi^{2}$ test) and all of these transitions accumulated in the context of Gp$\underline{\mathrm{CpX}}$ (Figure 2A). Numbers of the mutated TP53 nucleotide positions observed in the gastritis mucosa were identical to those that accumulated in human gastric cancer tissues (IARC TP53 database, R16, November 2012, http://p53.iarc.fr/), including codons 245 and 273 (Figure 2B, 2C).

\section{Mutation signature induced by AID activity that accumulated in human TP53 genes in vivo.}


The mutation spectrum determined in the TP53 sequences supported the possibility that AID-mediated deamination activity is deeply involved in the induction of driver mutations in tumor-related genes during human gastric carcinogenesis. To gain further insight into the role of AID in the emergence of somatic mutations in vivo, we determined the nucleotide sequences of the human TP53 genes in the gastric mucosa of the human TP53 knock-in (Hupki) mice ${ }^{33}$ after crossing them with AID transgenic (Tg) mice using deep sequencing analysis. Although the mean coverage reached 6434-fold, only one low-abundance mutation was detected in the human TP53 sequences from the gastric mucosa of 6 control Hupki mice with any type of codon 72 polymorphic variant (codon72 Arg/Arg, Pro/Pro and Arg/Pro) (Table 4). In contrast, low-abundance mutations accumulated in the human TP53 gene sequences in the gastric mucosa of 6 of $7(85.7 \%)$ Hupki/AID-Tg mice ( $\mathrm{p}=0.053$ by $\chi^{2}$ test, Table 4$) .8$ of $9(88.9 \%)$ genetic alterations determined in the gastric mucosa of 6 Hupki/AID-Tg mice were C:G>T:A transitions at the Gp대 motif ( $\mathrm{p}<0.01$ by $\chi^{2}$ test). Moreover, numbers of the low-abundance TP53 mutations were identical to the amino acid positions altered in human gastric cancer tissues 34,35 (Figure 2D). These findings provide evidence that the AID-mediated cytidine deamination could be deeply involved in the nucleotide alterations of the human TP53 gene that accumulate in the gastric mucosa during gastric tumorigenesis. 


\section{Discussion}

Chronic inflammation plays a critical role as a background of cancer development ${ }^{16,36,37}$. Therefore, it is reasonable to assume that stepwise accumulation of genetic alterations that contribute to tumorigenesis occurs in chronically inflamed epithelial cells. Consistently, several studies have reported that genetic aberrations are frequently detected in non-tumorous inflamed epithelial tissues where the risk of cancer development is remarkably high ${ }^{38-40}$. For example, TP53 mutations are detected in the inflamed mucosa of the colonic epithelium of patients with inflammatory bowel disease ${ }^{41,42}$. A recent sequencing study on Barrett's esophagus revealed that the mutational profiles of Barrett's esophagus and esophageal adenocarcinoma are remarkably similar, suggesting that genetic alterations in the metaplasia-carcinoma sequence of Barrett's esophagus occur much earlier than the histologic changes of frank dysplasia ${ }^{40}$. In the present study, using a whole exome sequencing technique, we demonstrated that somatic mutations accumulated not only in tumors but also in the non-tumorous inflamed gastric mucosa infected with $H$ pylori.

Regarding the cancer tissues, whole exome sequencing determined a remarkable difference in the mutation signature between MSI and MSS tumors. Namely, MSS tumors possessed a relatively lower number of total mutations, including SNVs and indels, compared with the MSI tumor. Indeed, the 4 MSS gastric cancer samples had an average of 56 non-silent somatic mutations with few indels, while the MSI gastric cancer sample had 427 non-silent somatic mutations including a large number of indels, consistent with the previous reports ${ }^{3,6}$. Moreover, the frequency of mutations at each nucleotide position in the MSS cancers tended to be lower than that in the MSI tumor. Notably, the mutation signature in non-tumorous background gastric mucosa did not differ significantly between patients with MSS and MSI tumors. These findings suggest that the outstanding features of genetic instability characterized as MSS and MSI 
phenotypes were not evident in the premalignant condition of gastric mucosa underlying $H$ pylori infection and resultant chronic inflammation, and would only become evident in the tumor cells after malignant transformation. Since the allelic fraction is highly dependent on the cell purity in the tissue samples, we should pay attention to the possibility that the tumor or epithelial cell purity could cause differences in the mutation frequencies in each tissue specimen analyzed.

Whole exome sequencing also clarified that the somatic mutations in various genes were latently accumulated in the non-tumorous gastritis mucosa of patients with $H$ pylori infection. It is notable that the total number of mutated genes of the non-tumorous gastric mucosa was smaller than that of the matched tumor tissues and the majority of the mutations detected in gastritis mucosa were predicted to have no influence on cell behavior, suggesting that most of the mutations in gastritis mucosa were passenger mutations. To unveil whether mutations of driver genes were present in gastritis mucosa, we conducted deep sequencing of the selected target genes on $H$ pylori (+) gastric epithelium. The sensitivity of ultra-deep sequencing analysis is primarily limited by errors introduced during any process of the sample preparation and the sequencing reaction, including PCR amplification, cluster amplification, cycle sequencing, image analysis, and others; thus, it is a challenge to distinguish rare mutations from sequencing $\operatorname{artifacts}^{18}$. In the present study, we optimized the deep sequencing by defining a strict filter setting and confirmed that no nucleotide alterations over the cut-off were detected in the control lymphocytes or in normal gastric mucosa. Furthermore, we validated the results by repeating the deep sequencing using independent amplicons derived from the same patients, although errors cannot completely be ruled out. Through these strict validation processes, we reproducibly detected low-abundance somatic mutations in non-tumorous gastric mucosa with $H$ pylori infection. Interestingly, TP53 was the most frequently mutated gene in gastritis mucosa among the six tumor-related genes analyzed. Strikingly, numbers of the mutated TP53 nucleotide 
positions observed in gastritis mucosa were located at the nucleotide positions of the mutations that accumulated in human gastric cancer tissues. It is not known whether the low-abundance TP53 mutations latently present in gastric mucosa with $H$ pylori infection derive from the clinically-undetectable small tumor cells or untransformed epithelial cells under premalignant conditions. It is reasonable to assume, however, that the low-abundance TP53 mutations in the background gastric mucosa with $H$ pylori infection could be the source of gastric cancer cells.

Recent studies showed that the mutation signature that accumulates in tumor tissues provides the clue to identifying the cause of genetic alterations during tumor development ${ }^{22-27,43}$. It was shown that $\mathrm{C}: \mathrm{G}>\mathrm{T}: \mathrm{A}$ transitions at $\mathrm{Xp} \underline{\mathrm{C}} \mathrm{G}$ trinucleotides were especially prominent in many cancer types $^{27,44}$. Among the $\mathrm{C}: \mathrm{G}>\mathrm{T}: \mathrm{A}$ transitions, the footprint of AID is characterized by $\mathrm{C}: \mathrm{G}>\mathrm{T}: \mathrm{A}$ alterations that occur in Gp$\underline{\mathrm{p}} \mathrm{XX}$ or Ap$\underline{\mathrm{CpX}}$ sequences $^{22,28,29,45-47}$. In the current study, we found that the frequency of $\mathrm{C}: \mathrm{G}>\mathrm{T}: \mathrm{A}$ transitions was the highest pattern of SNVs accumulated in the whole exome sequences of all five cancer tissues examined. Interestingly, the $\mathrm{C}: \mathrm{G}>\mathrm{T}: \mathrm{A}$ mutations in gastric cancer genomes predominantly accumulated in the context of Gp$\underline{\mathrm{C}} \mathrm{X}$ or $\mathrm{Ap} \underline{\mathrm{C}} \mathrm{pX}$, suggesting the involvement of AID-mediated cytidine deamination in the induction of somatic mutations during gastric carcinogenesis.

We previously revealed a causal relationship between mutagenic AID activity and the accumulation of TP53 mutations during the development of human gastric cancers ${ }^{14}$. The present findings showing the detection of low-abundance TP53 mutations with the typical footprint of AID activity in inflamed human gastric mucosa further support the putative role of AID in the generation of genetic alterations in an inflammatory microenvironment of gastric mucosa with $H$ pylori infection. The differences in p53 gene sequences between humans and mice, however, made it difficult to confirm whether constitutive AID expression in gastric mucosa in vivo contributes to the emergence of TP53 mutations that are identical to those observed in human 
gastritis and gastric cancer tissues. It is well established that Hupki mice, in which exons 4-9 of human TP53 were knocked in the corresponding mouse exons, are useful models for studying human TP53 mutagenesis ${ }^{33,48,49}$. Therefore, in this study we performed deep sequencing analyses on human TP53 gene of gastric mucosa derived from Hupki mice with, and without, constitutive AID expression. AID expression in gastric mucosa resulted in the accumulation of low-abundance human TP53 mutations with the typical signature of AID-mediated cytidine deamination. Strikingly, numbers of the amino acid changes determined in AID-expressing gastric mucosa of Hupki mice accumulated at positions identical to those altered in human gastric cancers. Taken together, these findings strongly suggest that constitutive AID expression in gastric epithelium plays a critical role in the accumulation of human TP53 mutations during the development of gastric cancers (Figure 3). To clarify the role of AID in the accumulation of somatic mutations and tumorigenesis in the stomach in vivo, further analyses of aberrant AID expression and resultant Trp53 mutations in gastric inflamed mucosa are required using previously established mouse models with inflammation-associated gastric carcinogenesis ${ }^{50-52}$.

In summary, we demonstrated that various mutations were latently accumulated in the gastric mucosa in patients with $H$ pylori infection. The combination of whole exome sequencing with deep sequencing enabled us to unveil the landscape of mutations that latently accumulated in non-tumorous gastritis mucosa. Interestingly, the mutation signature not only in gastric cancer genomes but also in gastritis mucosa suggested that AID-mediated deamination events were predominant during the process of gastric carcinogenesis. In particularly, a causal relationship between AID activity and the accumulation of TP53 mutations was also validated by in vivo models. It is expected that uncovering the landscape of genetic alterations in gastritis mucosa will contribute to clarifying the mechanism of gastric cancer development. 


\section{Acknowledgments}

We thank A. Takaori-Kondo, K. Shimizu and C. Kakimoto for deep sequencing analyses, M. Hollstein for Hupki mice, T. Honjo for AID Tg mice, and K. Kinoshita for his helpful suggestion. 


\section{Figure legends}

Figure 1. Somatic mutations in tumor and non-tumorous gastric tissues determined by whole exome sequencing.

(A) Number of non-synonymous and synonymous mutations in each sample. C001-C004 had gastric cancers with MSS features, and C005 had gastric cancer with MSI features.

(B) Distribution of the mutation frequency determined in each sample (at a frequency of $\geq 10 \%$ of reads). The proportions of mutations whose frequencies were less than $20 \%$ of the total reads significantly differed between cancer tissues and non-cancerous tissues in C001, C003, C004, and C005 ( $\mathrm{p}<0.01$ by $\chi^{2}$ test).

(C) Mutation signature determined in one MSI and four MSS gastric cancer tissues. C:G>T:A transitions in MSS cancer samples were significantly enriched ( $\mathrm{p}<0.01$ by ANOVA).

(D) Sequence context of the C:G>T:A transitions in one MSI and four MSS gastric cancer tissues. GpCpX patterns in MSS cancer samples were significant enriched ( $\mathrm{p}<0.01$ by ANOVA). *MSI status was determined from gastric cancer tissue.

**T: tumor, NT: non-tumorous gastritis mucosa

Figure 2. Low-abundance mutations accumulated in TP53 genes in human gastritis mucosa and human TP53-knock-in mice stomach.

(A) Mutation signature of 19 low-abundance TP53 mutations in H. pylori-related gastritis mucosa of 34 patients with or without gastric cancer determined by deep sequencing. These low-abundance TP53 mutations included $15 \mathrm{C}: \mathrm{G}>\mathrm{T}$ :A transitions, 3 T:A>C:G transitions and 1 $\mathrm{C}: \mathrm{G}>\mathrm{A}: \mathrm{T}$ transversion. $\mathrm{C}: \mathrm{G}>\mathrm{T}: \mathrm{A}$ transitions were significantly enriched ( $\mathrm{p}<0.01$ by ANOVA). (B) Distribution of low-abundance TP53 mutations in human gastritis mucosa of 34 patients with or without gastric cancer identified by deep sequencing. 
(C) Distribution of TP53 mutations in human gastric cancers obtained from IARC TP53 database (http://p53.iarc.fr/).

(D) Distribution of low-abundance TP53 mutations in gastric mucosa of seven 12-month-old Hupki/AID-Tg mice.

Figure 3. Schematic summary for AID-mediated TP53 mutations and gastric cancer development.

$H$ pylori infection and inflammatory signals such as TNF- $\alpha$ induce aberrant expression of a DNA-mutator enzyme, AID, in gastric epithelial cells. AID-mediated mutagenesis of driver genes including TP53 could contribute to gastric carcinogenesis. 


\section{Reference}

1. Meyerson M, Gabriel S, Getz G. Advances in understanding cancer genomes through second-generation sequencing. Nature Reviews Genetics 2010;11:685-696.

2. Bozic I, Antal T, Ohtsuki H, et al. Accumulation of driver and passenger mutations during tumor progression. Proc Natl Acad Sci U S A 2010;107:18545-18550.

3. Wang K, Kan J, Yuen ST, et al. Exome sequencing identifies frequent mutation of ARID1A in molecular subtypes of gastric cancer. Nat Genet 2011;43:1219-1223.

4. Zang ZJ, Cutcutache I, Poon SL, et al. Exome sequencing of gastric adenocarcinoma identifies recurrent somatic mutations in cell adhesion and chromatin remodeling genes. Nat Genet 2012;44:570-574.

5. Nagarajan N, Bertrand D, Hillmer AM, et al. Whole-genome reconstruction and mutational signatures in gastric cancer. Genome Biol 2012;13:R115.

6. Yoon K, Lee S, Han TS, et al. Comprehensive genome- and transcriptome-wide analyses of mutations associated with microsatellite instability in Korean gastric cancers. Genome Res 2013;23:1109-1117.

7. Uemura N, Okamoto S, Yamamoto S, et al. Helicobacter pylori infection and the development of gastric cancer. N Engl J Med 2001;345:784-789.

8. Chiba T, Marusawa H, Seno H, et al. Mechanism for gastric cancer development by Helicobacter pylori infection. J Gastroenterol Hepatol 2008;23:1175-1181.

9. Polk DB, Peek RM, Jr. Helicobacter pylori: gastric cancer and beyond. Nat Rev Cancer 2010;10:403-414.

10. Aoi T, Marusawa H, Sato T, et al. Risk of subsequent development of gastric cancer in patients with previous gastric epithelial neoplasia. Gut 2006;55:588-589.

11. Gutierrez-Gonzalez L, Graham TA, Rodriguez-Justo M, et al. The clonal origins of 
dysplasia from intestinal metaplasia in the human stomach. Gastroenterology 2011;140:1251-1260 e1-6.

12. Touati E, Michel V, Thiberge JM, et al. Chronic Helicobacter pylori infections induce gastric mutations in mice. Gastroenterology 2003;124:1408-1419.

13. Jenks PJ, Jeremy AH, Robinson PA, et al. Long-term infection with Helicobacter felis and inactivation of the tumour suppressor gene p53 cumulatively enhance the gastric mutation frequency in Big Blue transgenic mice. J Pathol 2003;201:596-602.

14. Matsumoto Y, Marusawa H, Kinoshita K, et al. Helicobacter pylori infection triggers aberrant expression of activation-induced cytidine deaminase in gastric epithelium. Nat Med 2007;13:470-476.

15. Matsumoto Y, Marusawa H, Kinoshita K, et al. Up-regulation of activation-induced cytidine deaminase causes genetic aberrations at the CDKN2b-CDKN2a in gastric cancer. Gastroenterology 2010;139:1984-1994.

16. Chiba $\mathrm{T}$, Marusawa $\mathrm{H}$, Ushijima $\mathrm{T}$. Inflammation-associated cancer development in digestive organs: mechanisms and roles for genetic and epigenetic modulation. Gastroenterology 2012;143:550-563.

17. Nagata N, Akiyama J, Marusawa H, et al. Enhanced expression of activation-induced cytidine deaminase in human gastric mucosa infected by Helicobacter pylori and its decrease following eradication. J Gastroenterol 2014; e-pub ahead of print 18 April 2013; doi: 10.1007/s00535-013-0808-Z.

18. Schmitt MW, Kennedy SR, Salk JJ, et al. Detection of ultra-rare mutations by next-generation sequencing. Proc Natl Acad Sci U S A 2012;109:14508-14513.

19. Nasu A, Marusawa H, Ueda Y, et al. Genetic heterogeneity of hepatitis $\mathrm{C}$ virus in association with antiviral therapy determined by ultra-deep sequencing. PLoS One 
2011;6:e24907.

20. Nishijima N, Marusawa H, Ueda Y, et al. Dynamics of hepatitis B virus quasispecies in association with nucleos(t)ide analogue treatment determined by ultra-deep sequencing. PLoS One 2012;7:e35052.

21. Sato Y, Yoshizato T, Shiraishi Y, et al. Integrated molecular analysis of clear-cell renal cell carcinoma. Nat Genet 2013;45:860-867.

22. Schmitz KM, Petersen-Mahrt SK. AIDing the immune system-DIAbolic in cancer. Semin Immunol 2012;24:241-245.

23. Nik-Zainal S, Alexandrov LB, Wedge DC, et al. Mutational processes molding the genomes of 21 breast cancers. Cell 2012;149:979-993.

24. Stephens PJ, Tarpey PS, Davies H, et al. The landscape of cancer genes and mutational processes in breast cancer. Nature 2012;486:400-404.

25. Roberts SA, Lawrence MS, Klimczak LJ, et al. An APOBEC cytidine deaminase mutagenesis pattern is widespread in human cancers. Nat Genet 2013;45:970-976.

26. Burns MB, Temiz NA, Harris RS. Evidence for APOBEC3B mutagenesis in multiple human cancers. Nat Genet 2013;45:977-983.

27. Alexandrov LB, Nik-Zainal S, Wedge DC, et al. Signatures of mutational processes in human cancer. Nature 2013;500:415-421.

28. Bransteitter R, Pham P, Calabrese P, et al. Biochemical analysis of hypermutational targeting by wild type and mutant activation-induced cytidine deaminase. J Biol Chem 2004;279:51612-51621.

29. Pham P, Bransteitter R, Petruska J, et al. Processive AID-catalysed cytosine deamination on single-stranded DNA simulates somatic hypermutation. Nature 2003;424:103-107.

30. Beale RC, Petersen-Mahrt SK, Watt IN, et al. Comparison of the differential 
context-dependence of DNA deamination by APOBEC enzymes: correlation with mutation spectra in vivo. J Mol Biol 2004;337:585-596.

31. Burns MB, Lackey L, Carpenter MA, et al. APOBEC3B is an enzymatic source of mutation in breast cancer. Nature 2013;494:366-370.

32. Kumar P, Henikoff S, Ng PC. Predicting the effects of coding non-synonymous variants on protein function using the SIFT algorithm. Nat Protoc 2009;4:1073-1081.

33. Besaratinia A, Pfeifer GP. Applications of the human p53 knock-in (Hupki) mouse model for human carcinogen testing. FASEB J 2010;24:2612-2619.

34. Petitjean A, Mathe E, Kato S, et al. Impact of mutant p53 functional properties on TP53 mutation patterns and tumor phenotype: lessons from recent developments in the IARC TP53 database. Hum Mutat 2007;28:622-629.

35. Olivier M, Hollstein M, Hainaut P. TP53 mutations in human cancers: origins, consequences, and clinical use. Cold Spring Harb Perspect Biol 2010;2:a001008.

36. Shimizu T, Marusawa H, Endo Y, et al. Inflammation-mediated genomic instability: roles of activation-induced cytidine deaminase in carcinogenesis. Cancer Sci 2012;103:1201-1206.

37. Grivennikov SI, Greten FR, Karin M. Immunity, inflammation, and cancer. Cell 2010;140:883-899.

38. Kou T, Marusawa H, Kinoshita K, et al. Expression of activation-induced cytidine deaminase in human hepatocytes during hepatocarcinogenesis. Int $\mathbf{J}$ Cancer 2007; 120:469-476.

39. Barrett MT, Sanchez CA, Prevo LJ, et al. Evolution of neoplastic cell lineages in Barrett oesophagus. Nat Genet 1999;22:106-109.

40. Streppel MM, Lata S, Delabastide M, et al. Next-generation sequencing of endoscopic 
biopsies identifies ARID1A as a tumor-suppressor gene in Barrett's esophagus. Oncogene 2014;33:347-357.

41. Yin J, Harpaz N, Tong Y, et al. p53 point mutations in dysplastic and cancerous ulcerative colitis lesions. Gastroenterology 1993;104:1633-1639.

42. Kern SE, Redston M, Seymour AB, et al. Molecular genetic profiles of colitis-associated neoplasms. Gastroenterology 1994;107:420-428.

43. Pleasance ED, Stephens PJ, O'Meara S, et al. A small-cell lung cancer genome with complex signatures of tobacco exposure. Nature 2010;463:184-190.

44. Lawrence MS, Stojanov P, Polak P, et al. Mutational heterogeneity in cancer and the search for new cancer-associated genes. Nature 2013;499:214-218.

45. Muramatsu M, Kinoshita K, Fagarasan S, et al. Class switch recombination and hypermutation require activation-induced cytidine deaminase (AID), a potential RNA editing enzyme. Cell 2000;102:553-563.

46. Cascalho M. Advantages and disadvantages of cytidine deamination. J Immunol 2004;172:6513-6518.

47. Liu M, Schatz DG. Balancing AID and DNA repair during somatic hypermutation. Trends Immunol 2009;30:173-181.

48. Luo JL, Yang Q, Tong WM, et al. Knock-in mice with a chimeric human/murine p53 gene develop normally and show wild-type p53 responses to DNA damaging agents: a new biomedical research tool. Oncogene 2001;20:320-328.

49. Kucab JE, Phillips DH, Arlt VM. Linking environmental carcinogen exposure to TP53 mutations in human tumours using the human TP53 knock-in (Hupki) mouse model. FEBS J 2010;277:2567-2583.

50. Tye H, Kennedy CL, Najdovska M, et al. STAT3-driven upregulation of TLR2 promotes 
gastric tumorigenesis independent of tumor inflammation. Cancer Cell 2012;22:466-478.

51. Oshima H, Oshima M. Mouse models of gastric tumors: Wnt activation and PGE2 induction. Pathol Int 2010;60:599-607.

52. Okumura T, Ericksen RE, Takaishi S, et al. K-ras mutation targeted to gastric tissue progenitor cells results in chronic inflammation, an altered microenvironment, and progression to intraepithelial neoplasia. Cancer Res 2010;70:8435-8445.

Author names in bold designate shared co-first authorship. 
Figure 1. Shimizu et al.

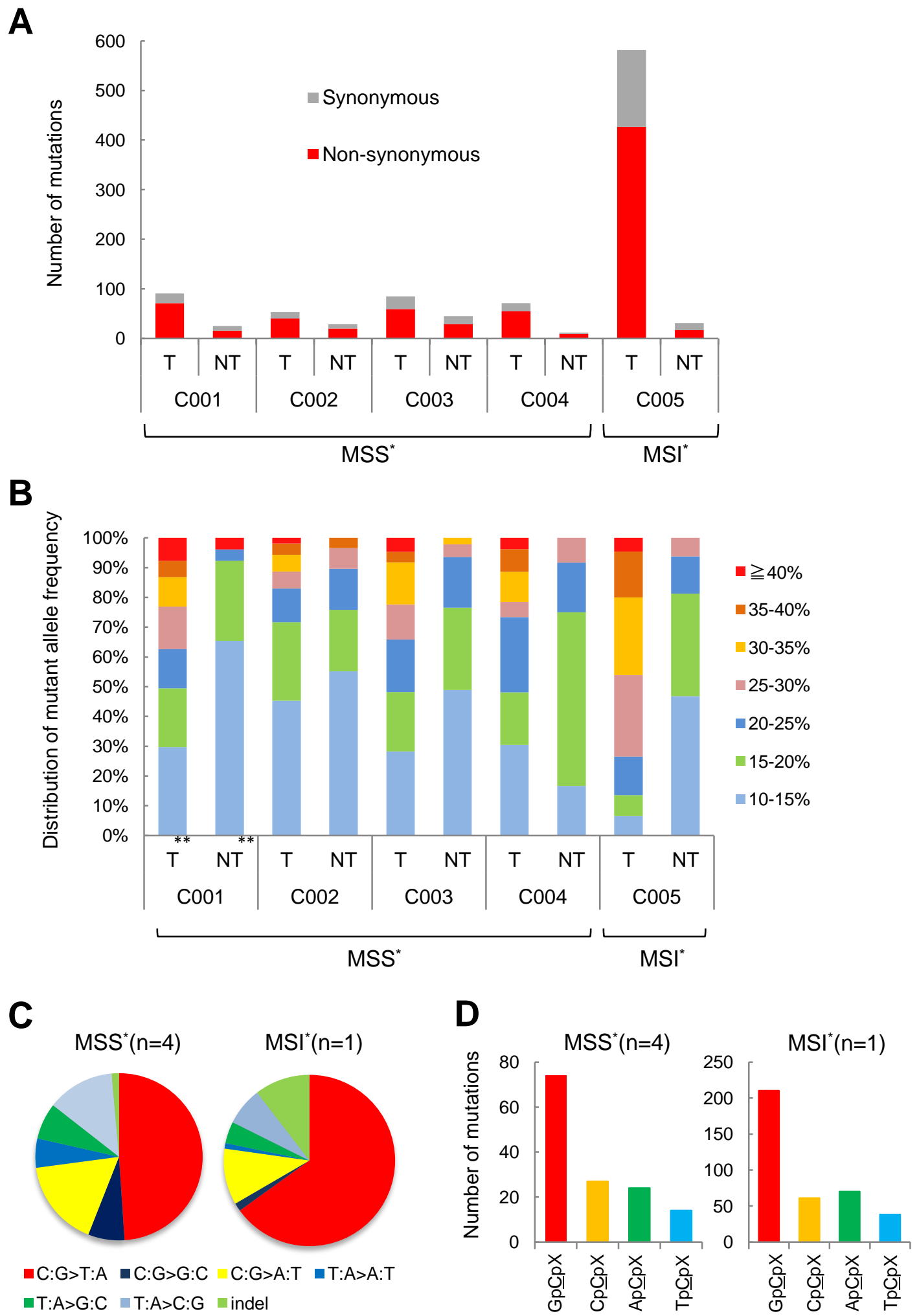


Figure 2. Shimizu et al.

A

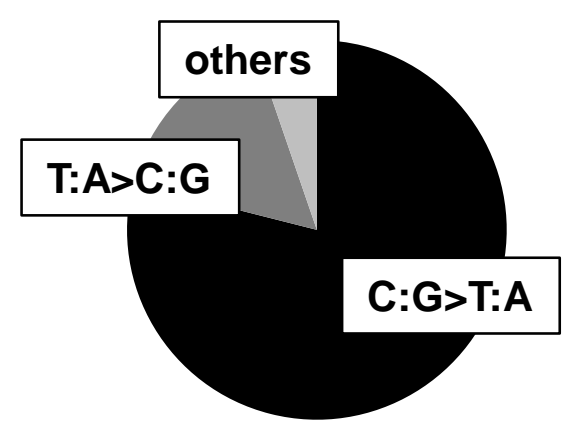

B

Human $\boldsymbol{H}$ pylori-infected gastritis

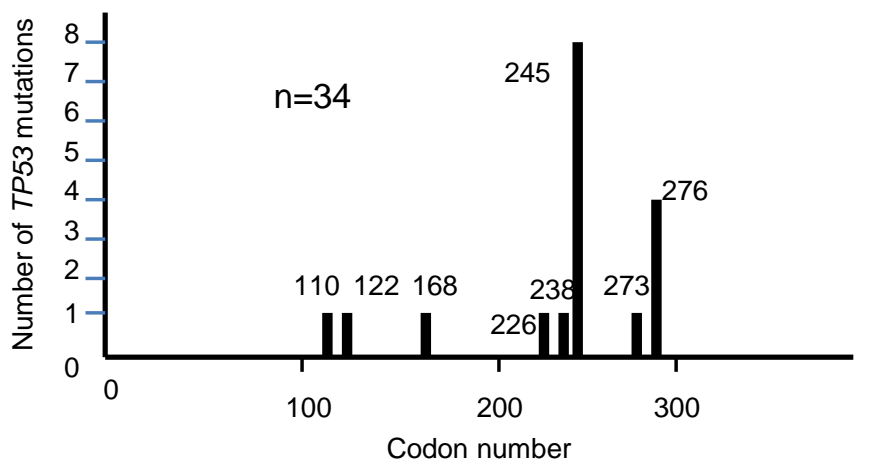

C

Human gastric cancers

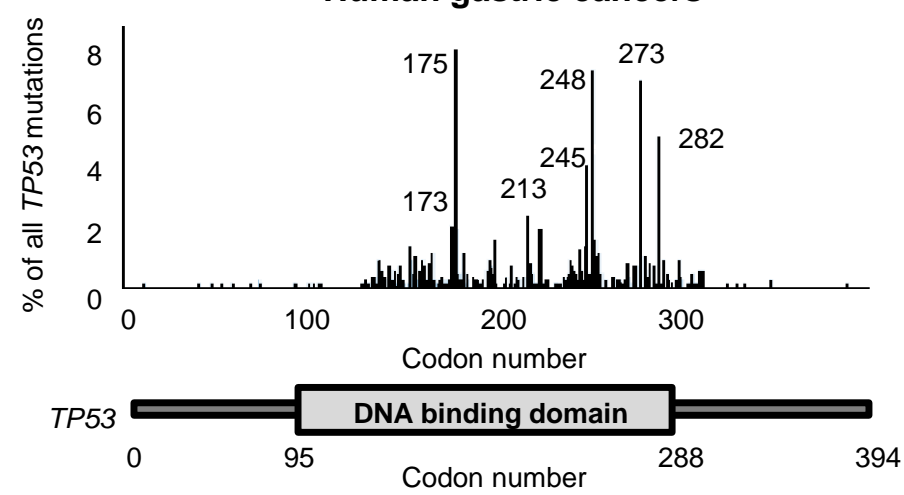

D

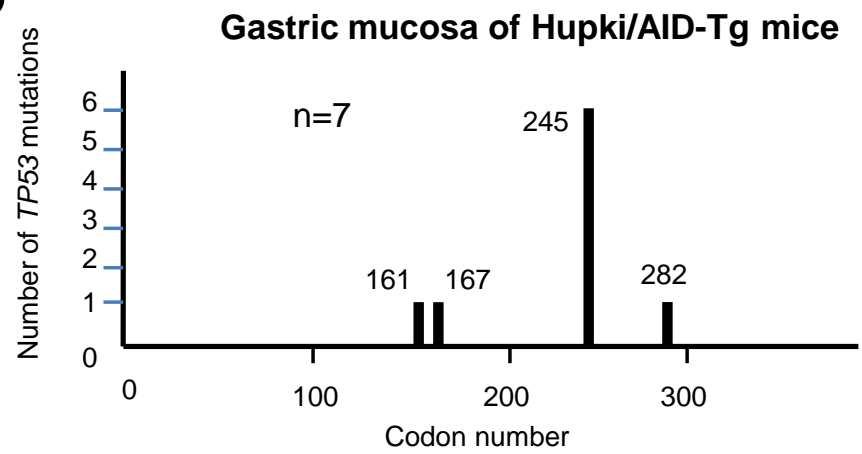




\section{Figure 3. Shimizu et al.}

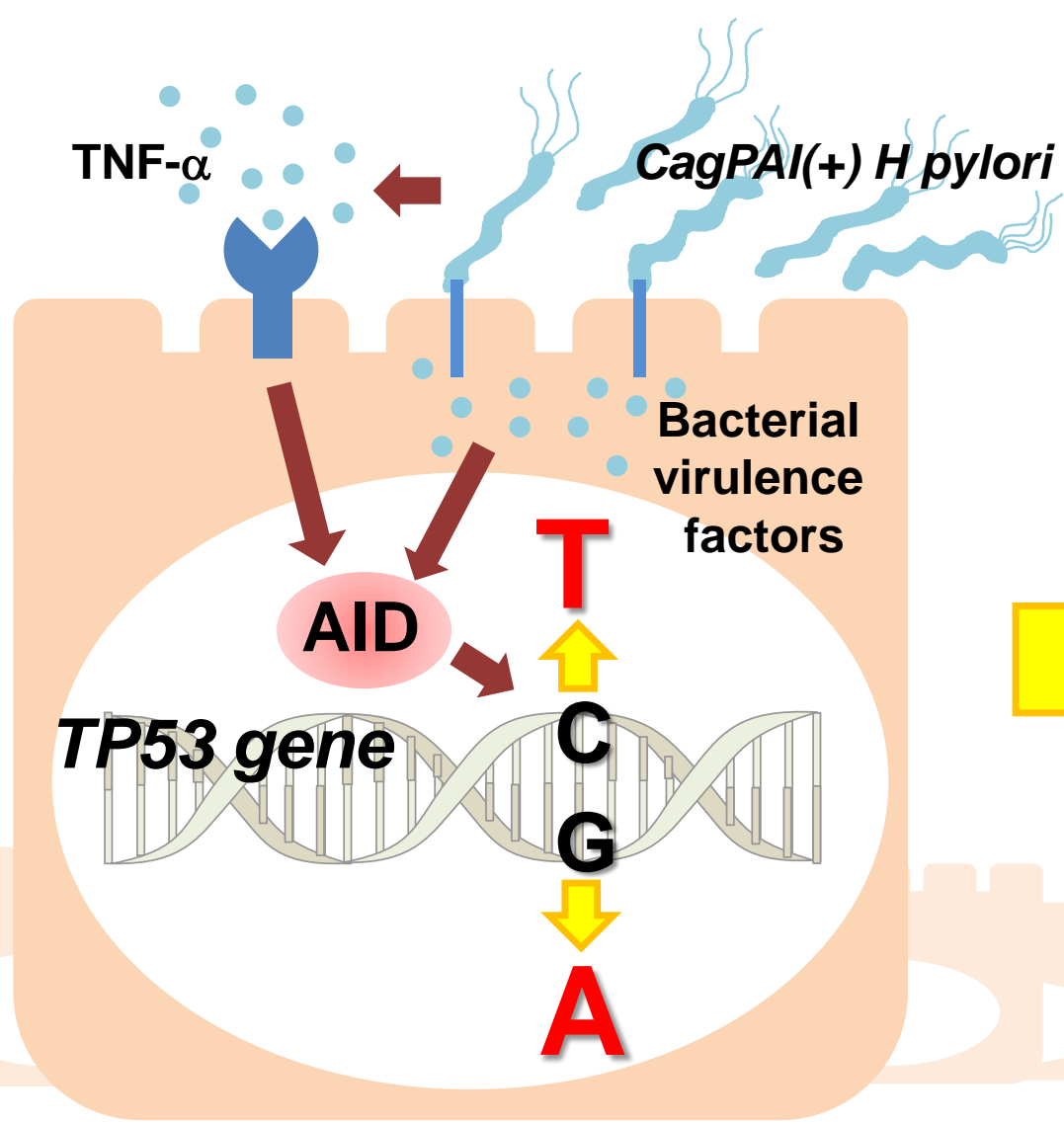

Chronic Gastritis

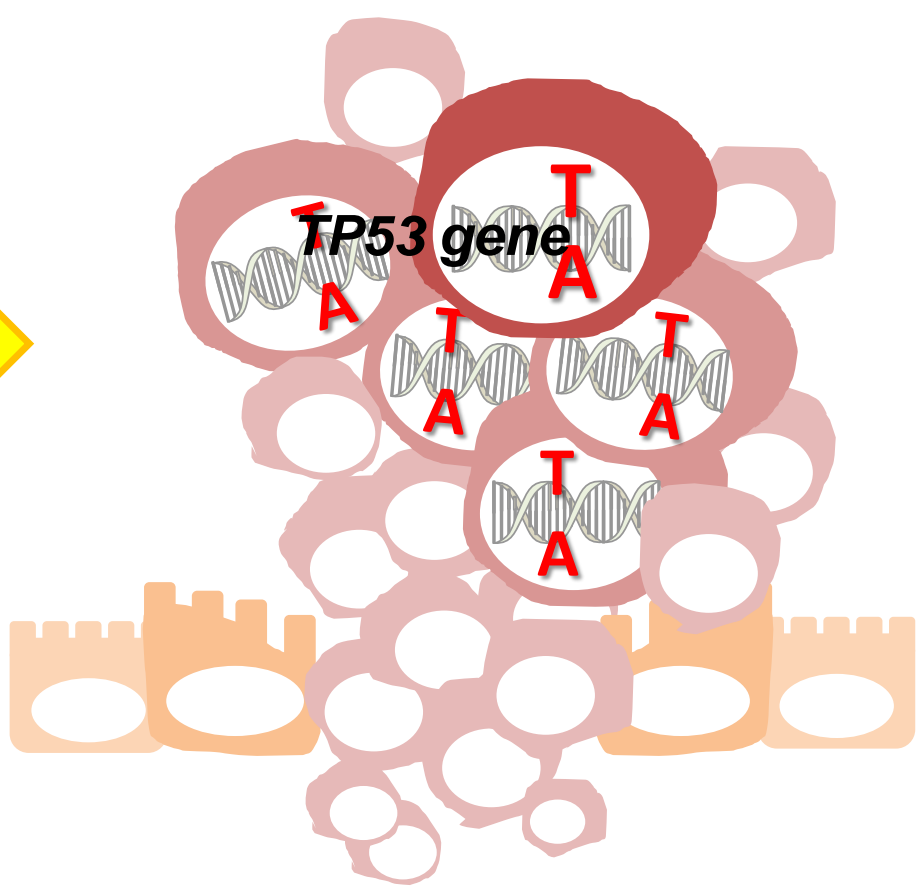

Gastric Cancer 
Table 1. List of 26 genes recurrently mutated in two or more tumor tissues of 5 gastric cancer patients.

\begin{tabular}{|c|c|c|c|c|}
\hline Gene symbol & Gene name & $\begin{array}{l}\text { Gene } \\
\text { size } \\
\text { (bp) }\end{array}$ & $\begin{array}{c}\begin{array}{c}\text { \# of } \\
\text { mutated } \\
\text { samples }\end{array}\end{array}$ & $\begin{array}{c}\text { \# of } \\
\text { mutations }\end{array}$ \\
\hline OBSCN & $\begin{array}{l}\text { obscurin, cytoskeletal calmodulin and titin-interacting } \\
\text { RhoGEF }\end{array}$ & 23,907 & 3 & 4 \\
\hline TP53 & tumor protein $\mathrm{p} 53$ & 1,182 & 3 & 3 \\
\hline MUC17 & mucin 17 , cell surface associated & 13,482 & 2 & 2 \\
\hline HRNR & hornerin & 8,553 & 2 & 3 \\
\hline$F L G$ & filaggrin & 12,186 & 2 & 2 \\
\hline PCDHB5 & protocadherin beta 5 & 2,388 & 2 & 3 \\
\hline PRB1 & proline-rich protein BstNI subfamily 1 & 996 & 2 & 2 \\
\hline$R O R B$ & RAR-related orphan receptor B & 1,380 & 2 & 2 \\
\hline SLC35G6 & solute carrier family 35, member G6 & 1,017 & 2 & 2 \\
\hline ARID1A & AT rich interactive domain $1 \mathrm{~A}$ (SWI-like) & 6,858 & 2 & 2 \\
\hline CACNA1B & $\begin{array}{l}\text { calcium channel, voltage-dependent, } \mathrm{N} \text { type, alpha 1B } \\
\text { subunit }\end{array}$ & 7,020 & 2 & 2 \\
\hline CD163 & CD163 molecule & 3,471 & 2 & 2 \\
\hline CEP164 & centrosomal protein $164 \mathrm{kDa}$ & 4,383 & 2 & 2 \\
\hline CNGB1 & cyclic nucleotide gated channel beta 1 & 900 & 2 & 2 \\
\hline COL5A1 & collagen, type V, alpha 1 & 5,517 & 2 & 2 \\
\hline CYP2A6 & $\begin{array}{l}\text { cytochrome P450, family 2, subfamily A, polypeptide } \\
6\end{array}$ & 1,485 & 2 & 2 \\
\hline FCGBP & $\mathrm{Fc}$ fragment of $\mathrm{IgG}$ binding protein & 16,218 & 2 & 2 \\
\hline GPC6 & glypican 6 & 1,668 & 2 & 2 \\
\hline HCN2 & $\begin{array}{l}\text { hyperpolarization activated cyclic nucleotide-gated } \\
\text { potassium channel } 2\end{array}$ & 2,670 & 2 & 2 \\
\hline РСDHA3 & protocadherin alpha 3 & 2,853 & 2 & 2 \\
\hline RABGGTA & Rab geranylgeranyltransferase, alpha subunit & 1,704 & 2 & 2 \\
\hline RAD54L2 & RAD54-like 2 (S. cerevisiae) & 4,404 & 2 & 2 \\
\hline ROPN1B & rhophilin associated tail protein $1 \mathrm{~B}$ & 639 & 2 & 2 \\
\hline SPDYE5 & speedy homolog E5 (Xenopus laevis) & 1,014 & 2 & 2 \\
\hline SUPT6H & suppressor of Ty 6 homolog (S. cerevisiae) & 5,181 & 2 & 2 \\
\hline TUBGCP2 & tubulin, gamma complex associated protein 2 & 2,793 & 2 & 2 \\
\hline
\end{tabular}


Table 2. Low-abundance mutations in $\mathrm{H}$ pylori-related gastritis mucosa of patients with gastric cancer detected by deep sequencing.

\begin{tabular}{|c|c|c|c|c|c|c|}
\hline Case $^{*}$ & $\begin{array}{c}\text { TP53 DNA } \\
\text { binding domain }\end{array}$ & $\begin{array}{c}A R I D 1 A \\
\text { exon20 }\end{array}$ & $\begin{array}{c}M L L 2 \\
\text { SET domain }\end{array}$ & $\begin{array}{c}\text { SUV39H1 } \\
\text { SET domain }\end{array}$ & $\begin{array}{c}\text { CTNNB1 } \\
\text { exon3 }\end{array}$ & $\begin{array}{c}\text { PIK3CA } \\
\text { exon21 }\end{array}$ \\
\hline $\mathrm{C} 001$ & None & None & None & None & None & None \\
\hline $\mathrm{C} 002$ & $\begin{array}{l}\text { c.365T }>C \text { p.V122A } \\
\text { c.734G }>A \text { p.G245D }\end{array}$ & None & None & None & None & None \\
\hline $\mathrm{C} 003$ & None & c.5763C >T p.T1921T & None & None & None & None \\
\hline C004 & c.734G >A G245D & None & None & None & None & None \\
\hline $\mathrm{C} 005$ & None & None & None & None & None & None \\
\hline $\mathrm{C} 006$ & None & $\begin{array}{l}\text { c.5920C }>\text { T p.Q1974X } \\
\text { c.6001A }>\text { G p.M2001V }\end{array}$ & None & None & None & None \\
\hline $\mathrm{C} 007$ & c.712T>C p.C238R & None & None & None & None & None \\
\hline $\mathrm{C} 008$ & None & None & $\begin{array}{l}\text { c.16373A>G p.E5458G } \\
\text { c.16438A>G p.N5480D }\end{array}$ & None & None & None \\
\hline $\mathrm{C} 009$ & c.734G>A p.G245D & None & None & None & None & None \\
\hline $\mathrm{C} 010$ & None & None & None & None & None & None \\
\hline $\mathrm{C} 011$ & c.734G>A p.G245D & None & None & None & None & None \\
\hline $\mathrm{C} 012$ & c.329G $>T$ p.R110L & c.5983C $>$ T p.P1995S & None & None & None & None \\
\hline $\mathrm{C} 013$ & $\begin{array}{l}\text { c.503A }>\text { G p.H168R } \\
\text { c.827C }>\text { T p.A276V }\end{array}$ & None & None & None & None & None \\
\hline $\mathrm{C} 014$ & None & None & None & None & None & None \\
\hline $\mathrm{C} 015$ & None & $\begin{array}{l}\text { c. } 5307 \mathrm{~A}>\mathrm{G} \text { p.L1769L } \\
\text { c.5607G }>\text { A p.R1869R }\end{array}$ & None & None & None & None \\
\hline $\mathrm{C} 016$ & None & None & c. $16428 \mathrm{C}>\mathrm{T}$ p.S5476S & None & c. $99 \mathrm{~T}>\mathrm{C}$ p.S33S & None \\
\hline $\mathrm{C} 017$ & c.734G >A p.G245D & c. $5406 \mathrm{G}>$ A p.E1802E & None & None & None & None \\
\hline $\mathrm{C} 018$ & None & None & None & None & None & None \\
\hline $\mathrm{C} 019$ & None & None & None & None & None & None \\
\hline $\mathrm{C} 020$ & None & None & c.16379T >A p.V5460E & None & None & None \\
\hline $\mathrm{C} 021$ & None & None & None & None & None & None \\
\hline $\mathrm{C} 022$ & None & None & None & None & None & None \\
\hline $\mathrm{C} 023$ & c.817C $>\mathrm{T}$ p.R273C & None & None & None & None & None \\
\hline C024 & c.827C $>\mathrm{T}$ p.A276V & $\begin{array}{l}\text { c.5239C }>T \text { p.P1747S } \\
\text { c.5503C }>T \text { p.Q1835X }\end{array}$ & None & None & None & None \\
\hline $\mathrm{C} 025$ & None & c.5482T $>C$ p.S1828P & None & None & None & None \\
\hline $\mathrm{C} 026$ & c.734G>A p.G245D & None & None & None & None & None \\
\hline $\mathrm{C} 027$ & None & None & None & None & None & None \\
\hline $\mathrm{C} 028$ & None & None & None & None & None & None \\
\hline
\end{tabular}

All low-abundance mutations in this table were validated by repeated deep sequencing.

Low-abundance mutations written in bold letters are non-synonymous, and those written in small letters are synonymous

${ }^{*} \mathrm{C} 001-\mathrm{C} 028$ are $H$ pylori-related gastritis patients with gastric cancer. 
Table 3. Low-abundance mutations in $\boldsymbol{H}$ pylori-related gastritis mucosa of patients without gastric cancer and normal gastric mucosa detected by deep sequencing.

\begin{tabular}{|c|c|c|c|c|c|c|}
\hline Case $^{*}$ & $\begin{array}{c}\text { TP53 DNA } \\
\text { binding domain }\end{array}$ & $\begin{array}{c}A R I D 1 A \\
\text { exon20 }\end{array}$ & $\begin{array}{c}M L L 2 \\
\text { SET domain }\end{array}$ & $\begin{array}{c}\text { SUV39H1 } \\
\text { SET domain }\end{array}$ & $\begin{array}{l}C T N N B 1 \\
\text { exon3 }\end{array}$ & $\begin{array}{r}P I K 3 C A \\
\text { exon21 }\end{array}$ \\
\hline A029 & $\begin{array}{l}\text { c.734G }>\text { A p.G245D } \\
\text { c.827C }>\text { T p.A276V }\end{array}$ & None & None & c.1029G>A p.R343R & None & None \\
\hline $\mathrm{A} 030$ & $\begin{array}{l}\text { c.412G }>\text { A p.A138T } \\
\text { c.677G }>\text { A p.G226D }\end{array}$ & None & None & None & None & None \\
\hline A031 & c.827C $>$ T p.A276V & None & None & None & None & None \\
\hline A032 & c.734G >A p.G245D & None & None & None & None & None \\
\hline A033 & None & None & None & None & None & None \\
\hline A034 & None & c.5983C $>$ T p.P1995S & None & None & None & None \\
\hline N035 & None & None & None & None & None & None \\
\hline N036 & None & None & None & None & None & None \\
\hline N037 & None & None & None & None & None & None \\
\hline N038 & None & None & None & None & None & None \\
\hline N039 & None & None & None & None & None & None \\
\hline
\end{tabular}

All low-abundance mutations in this table were validated by repeated deep sequencing.

Low-abundance mutations written in bold letters are non-synonymous, and those written in small letters are synonymous

*A029-A034 are $H$ pylori-related gastritis patients without gastric cancer. N035-N039 are individuals without $H$ pylori infection and gastric cancer. 
Table 4. Low-abundance TP53 mutations in gastric mucosa of Hupki/AID-Tg mice detected by deep sequencing.

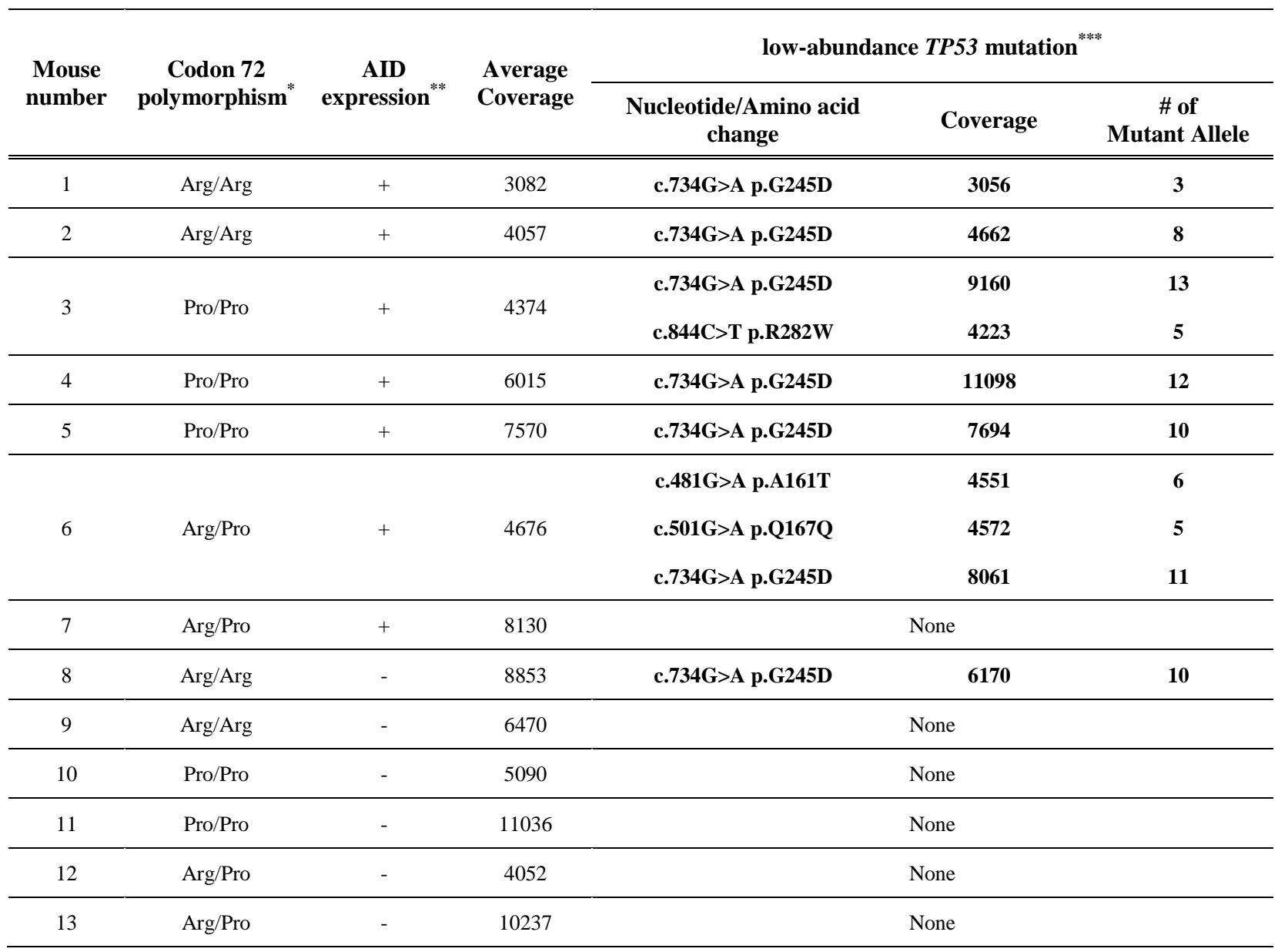

All mice were male and sacrificed at 12 months of age.

*Hupki mouse strain with arginine(Arg)/Arg, proline(Pro)/Pro or Arg/Pro variants at codon 72 of human TP53. A common polymorphism is frequently observed at codon 72 in TP53, resulting in either a Pro or an Arg, and the polymorphism at codon 72 affect critical biochemical properties of p53 protein (Ref. 49)

**Constitutive AID expression of the stomach was achieved by crossing Hupki mice with AID-Tg mice.

*** Low-abundance somatic mutations accumulated in human TP53 gene sequences in the gastric mucosa of Hupki mice. 